TecnoX 4.0

Ética, derechos humanos y tecnologías libres

\title{
Aprendizaje inmersivo para una educación basada en la empatía: "aulas sin fronteras", sobre un modelo de open-science-data a través del arte en realidad aumentada.
}

David Chaupis-Meza, Nicolas Kouri, Jean Pierre Tincopa, Dulce E. Alarcón-Yaquetto.

\section{RESUMEN}

KOHLI, A. (2003):

“...democracy as a form of government, democratization of power as a political process, and socio-economic democracy as a possible goal, are analytically and empirically distinguishable; there is no linear or automatic trend from a democratic government to democratization of power and to a more egalitarian democracy".

¿Cómo podría la democratización del conocimiento impactar en la educación de la ciudadanía? ¿Qué herramientas tecnológicas son las necesarias para alcanzar tal revolución educativa en Latinoamérica? ¿Necesitamos acaso una educación disruptiva? Aunque existen diversos métodos para la innovación educativa, que bien podrían vincularse hacia una revolución educativa, pocos son los que favorecen al pensamiento divergente. Algunos métodos podrían ser: a) el aprendizaje basado en proyectos; b) la gamificación; c) la clase al revés; d) el design thinking; e) el design for change; f) el aprendizaje cooperativo; entre otros.

Sin embargo, existen aún altos índices de desmotivación, desinterés y hasta fracaso escolar que se ve reflejado en la pérdida del asombro por el conocimiento per se. Por lo tanto: ¿qué está fallando en el actual sistema pedagógico?, tal parece ser, que estos son los retos para la formación del ciudadano del siglo XXI. Retos que alienten a la integración desde la inclusión social, la inteligencia colectiva, la valoración de la educación emocional, fomentando la interdisciplinariedad, en la exploración de nuevos medios de comunicación. Todo esto nos lleva a una educación basada en la empatía.

En tal sentido, HyperLab (un laboratorio para la exploración cibernética) propone un método dinámico de aprendizaje inmersivo a partir del arte integrado con las tecnologías libres (como la visualización de data analógica y la realidad aumentada). El resultado del desarrollo de estas aplicaciones tecnológicas, en conjunción con el arte callejero (como el graffiti y/o el mural), estimularían la empatía hacía una enseñanza intuitiva; por ende, planteamos la generación de "aulas sin fronteras", como espacios públicos co-creativos para la comprensión intuitiva en temas de ciencias. Todo lo que imaginas, existe.

Palabras claves: empatía, educación popular, innovación social, tecnologías abiertas, arte callejero. 